\title{
Transmit Diversity versus Beamforming System in Correlated Fading Channel
}

\author{
Abdul W. Umrani ${ }^{1}$, Yong L. Guan ${ }^{2}$, Fahim A. Umrani ${ }^{1}$ \\ ${ }^{1}$ Institute of Information and Communication Technologies, Mehran University of Engineering \& Technology, \\ Jamshoro, Pakistan \\ ${ }^{2}$ Division of Communication Engineering, School of EEE, Nanyang Technological University, Singapore City, Singapore \\ E-mail: waheed.umrani@faculty.muet.edu.pk \\ Received May 25, 2010; revised June 27, 2010; accepted July 31, 2010
}

\begin{abstract}
The performance analysis, using outage capacity for a forward link cellular communications system is presented. The effects of correlated fading, the distribution of angle of arrivals, and the array configurations are considered. The objective is to examine the effect of angle of arrival (AOA) energy distributions on two systems (transmit diversity and beamforming) with angle spread. We do so by comparing the performance of transmit diversity with a system that uses beamforming to point a relatively narrow beam at the mobile station. Analytical results show that the transmit diversity system using uniform linear arrays (ULA) and uniform circular arrays (UCA) with truncated Laplacian AOA, performs better even at smaller angle spreads as compared to other energy distributions. The ULA geometry is a preferable configuration for transmit diversity system as compared to UCA.
\end{abstract}

Keywords: Antenna Arrays, Angle Spread, Fading Correlation, Diversity Performance, Fading Channels, Cellular Radio

\section{Introduction}

The use of multiple antennas at the base station to create spatial diversity provides an effective technique for reducing the effects of fading. It is known that Transmit diversity system provides the benefit of diversity with no array gain, while the transmit beamforming provides array gain but no diversity [1]. The beamforming system is normally designed so that the fading at the antennas is highly correlated for wide range of angular spreads, while transmit diversity is designed so that the fading will be decorrelated for small angle spreads. Transmit diversity systems are typically optimal in rich scattering environments existing in indoor environments, which means that the fading between antenna elements are independent. However, in low scattering environments (e.g. in line-of-sight) where the fading is correlated, these systems do not perform well. The authors are interested in the performance of both systems as the fading correlation changes from perfectly correlated to completely independent with AOA energy distribution under consideration (i.e., Uniform, truncated Gaussian, and truncated Laplacian).
Some of the research works using antenna arrays in the downlink for particular environments have been proposed in [2-6] for correlated fading channels. In these papers, the authors use combination of space-time block codes (STBC) with beamforming. In $[4,5]$, the authors, have studied the impact of fading correlation on the performance of STBC. In [6], it was shown that the SNR for both the transmit diversity method and beamformer is a function of the sum of eigenvalues of fading correlation matrix. Since fading correlation matrix is also a function of angle of arrival, with some distribution, depending on the particular environment. Some measurement results show that the AOA energy distribution in general has a shape which more closely resembles Gaussian or Laplacian distribution [7], while other experiments suggest the use of truncated Gaussian AOA distribution, when the base station is near to the mobile station and truncated Laplacian AOA distribution for micro-cellular radio environments.

The authors in [8], presented a comparative analysis and the tradeoffs between transmit diversity systems and beamforming for different number of antennas for the forward link cellular communication system with and without hand-off. However, the effect of angle of arrival 
(AOA) distributions and the array configuration (i.e., linear, circular, etc.) were not taken into account in [8]. In this paper, we study the transmit diversity and transmit beamforming for multi-input-single-output (MISO) system, and present a comparative analysis/results of the two systems by taking into account the various AOA energy distributions, and array geometry. The objective is to examine the effect of angle of arrival (AOA) distributions on two systems with angle spread, which is defined as standard deviation of the distribution under consideration (i.e., Uniform, truncated Gaussian, and truncated Laplacian). We also want to see the performance of uniform linear arrays (ULA) and uniform circular arrays (UCA) with these distributions. In this paper, frequency division duplex (FDD) system is considered, where the up and downlink channels fade independently. Therefore, the base station (BS) is unable to estimate the downlink channel. This is also referred to as open-loop transmit diversity. Certainly, the closed-loop transmit diversity, which uses feedback, has the potential to provide diversity as well as array gain advantage, but that is beyond the scope of this paper. We also assume that the receiver knows the channel by using pilot or training signal. The part of this work is also reported in $[9,10]$.

In Section 2, we present a detail description of system model. Then a brief discussion of spatial correlation and outage capacity is presented in Section 3. Section 4 details the results and some discussion on it. Finally, conclusion is presented in Section 5.

Notation: Lower case boldface letters are used to denote vectors and upper case boldface letters to denote matrices, (. $)^{\mathrm{T}}$ denotes the transpose, and $(.)^{\mathrm{H}}$ denotes the Hermitian transpose. In addition, $\mathfrak{R}(\mathbf{A})$ means the real part of $\mathbf{A}, \mathfrak{I}(\mathbf{A})$ means the imaginary part of $\mathbf{A}$.

\section{System Model}

A wireless communication system with $M$ array antennas (ULA or UCA) at the base station and a single antenna at the mobile station is considered. Specifically, the ULA consists of four elements located on the $x$ direction while the UCA consists of four elements lying on a circle about the origin as shown in the Figure $\mathbf{1}$ below. This configuration is similar to as considered by [11] (Figure 1).

Consider an array receiving a narrowband signal from a point source. Let the array response vector be denoted by $\mathbf{a}(\theta)=\left[a_{1}, \ldots, a_{M}\right]^{\mathrm{T}}$, where $\theta$ is the direction of the source relative to array. In the case of ULA, it is given by

$$
a_{m}(\theta)=e^{j 2 \pi(m-1)(d / \lambda) \sin \theta}
$$

where $a_{m}(\theta)$ is the $m$ th entry a $(\theta), d$ is the spacing between the antennas, $\lambda$ is the wavelength. Without loss of generality, we assumed here that the phase of first element is zero. Note that adding the same fixed phase to all

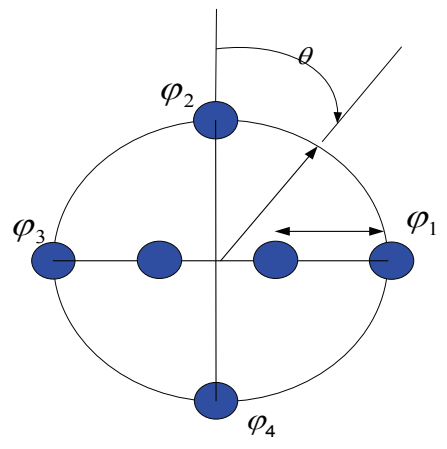

Figure 1. Four element ULA and UCA antenna array configuration at the base station. $R$ is radius of circular array and $d$ is the spacing between array elements, $d=2 R / 3$.

of the elements of the array does not change any of the results. Similarly, in the case of UCA, it is given by,

$$
a_{m}(\theta)=e^{j 2 \pi(m-1)(R / \lambda) \sin (\xi) \cos \left(\theta-\varphi_{m}\right)}
$$

where $R$ is the circular radius of the array, $\xi$ is the elevation angle of arrival. For simplicity, only azimuth angles are considered in propagation geometry (i.e., $\xi=90^{\circ}$ ), and $\varphi_{m}$ is the angle that each element location makes with horizontal axis as shown in [11] Figure 1. For four-element UCA configuration, $\varphi_{m}$ 's are $0^{\circ}, 90^{\circ}, 270^{\circ}$ and $360^{\circ}$, respectively.

Let $S N R$ denote the average signal-to-noise ratio at the mobile station, when the base station uses a single antenna. Then, the instantaneous signal-to-noise ratio $(s n r)$ observed by ideal transmit diversity system is given by [8],

$$
s n r=\frac{S N R}{2 M} \gamma
$$

where

$$
\gamma=2 \sum_{m=1}^{M}\left|a_{m}\right|^{2}=2 \mathbf{a}^{H} \mathbf{a}=2 \mathbf{u}^{H} \mathbf{R u}
$$

where $\mathbf{u}$ is vector of independent Gaussian random variables with zero mean and unit variance, $\mathbf{R}$ is the spatial covariance matrix of $\mathbf{a}$. Using singular value decomposition of $\mathbf{R}, \gamma$ can finally be written as

$$
\gamma=2 \sum_{m=1}^{M} \lambda_{m}\left|u_{m}\right|^{2}
$$

where $\lambda_{m}$ are singular values of $\mathbf{R}$. In general, the pdf of $\gamma$ is given by [12]

$$
f(\gamma)=\sum_{m=1}^{M} \alpha_{m} p_{m}(\gamma)
$$

where

$$
p_{m}(\gamma)=\frac{1}{\lambda_{m}} e^{-\gamma / \lambda_{m}}, \quad \& \quad \alpha_{m}=\prod_{i=1, i \neq m}^{M} \frac{\lambda_{m}}{\lambda_{m}-\lambda_{i}}
$$




\section{Spatial Correlation and Outage Probability}

In [13], a detail analysis on the spatial fading correlation for uniform AOA on linear arrays was carefully studied. Further research on spatial fading correlation has been carried out for various AOA energy distributions in [11, 14-18], which include Gaussian and Laplacian distribution etc. In [11], the bit error rate performance is computed for ULA and UCA for truncated Gaussian AOA distribution, and in [14], a correct version of closed form equation for spatial fading correlation matrix is reported. Similarly, for truncated Laplacian distribution the fading correlation equations are reported in $[16,17]$. Some measurement results suggest that the AOA energy distribution in general has a shape which more closely resembles Gaussian or Laplacian distribution [7], while other experiments suggest the use of truncated Gaussian AOA distribution, when the base station is near to the mobile and truncated Laplacian AOA distribution for microcellular radio environments.

For the sake of completeness we write the truncated Gaussian AOA distribution [11], but corrected in [14] as,

$$
f\left(\zeta_{s}\right)=C_{g} e^{-\frac{\left(\zeta_{s}-\theta\right)^{2}}{2 \sigma_{a}^{2}}} \quad-\pi+\theta \leq \zeta_{s} \leq \pi+\theta
$$

where

$$
C_{g}=\frac{1}{\operatorname{erf}\left(\frac{\pi}{\sqrt{2} \sigma_{a}}\right) \sqrt{2 \pi} \sigma_{a}}
$$

Similarly truncated Laplacian AOA distribution is written as [16],

$$
f(\zeta)=C_{l} e^{-s|\theta-\zeta|}-\pi+\theta \leq \zeta \leq \pi+\theta
$$

where

$$
C_{l}=\frac{s}{2\left(1-e^{-a \pi}\right)}
$$

In (8)-(11), $\theta$ is the mean AOA, $\sigma_{a}$ is the angle spread of truncated Gaussian distribution. Similarly, $s$ is the decay factor, which is related to the angle spread. Specifically, as $s$ increases, the angle spread decreases. The closed form expressions for the real and imaginary parts of spatial covariance matrix $\mathbf{R}$, for uniform linear array having uniform AOA, can easily be computed from [13]. For the case of truncated Gaussian AOA, the real and imaginary parts of spatial covariance matrix for ULA, and UCA are given [11], and modified as in [14] by (12) -(15), respectively.

$$
\begin{aligned}
\mathfrak{R}\{\mathbf{R}(m, n)\}= & J_{0}\left(Z_{l}\right)+2 \sqrt{2 \pi} C_{g} \sigma_{a} . \\
& \sum_{k=1}^{\infty} e^{-2 k^{2} \sigma_{a}^{2}} J_{2 k}\left(Z_{l}\right) \cos [2 k(\theta)]
\end{aligned}
$$

$$
\begin{aligned}
& \mathfrak{J}\{\mathbf{R}(m, n)\}= 2 \sqrt{2 \pi} C_{g} \sigma_{a} \sum_{k=0}^{\infty} e^{-\frac{(2 k+1)^{2} \sigma_{a}^{2}}{2}} J_{2 k+1}\left(Z_{l}\right) . \\
& \sin [(2 k+1)(\theta)] \\
& \mathfrak{R}\{\mathbf{R}(m, n)\}= J_{0}\left(Z_{c}\right)+2 \sqrt{2 \pi} C_{g} \sigma_{a} \cdot \\
& \sum_{k=1}^{\infty} e^{-2 k^{2} \sigma_{a}^{2}} J_{2 k}\left(Z_{c}\right) \cos [2 k(\theta+\alpha)] \\
& \mathfrak{J}\{\mathbf{R}(m, n)\}= 2 \sqrt{2 \pi} C_{g} \sigma_{a} \sum_{k=0}^{\infty} e^{-\frac{(2 k+1)^{2} \sigma_{a}^{2}}{2}} J_{2 k+1}\left(Z_{c}\right) . \\
& \sin [(2 k+1)(\theta+\alpha)]
\end{aligned}
$$

Similarly, for the case of truncated Laplacian AOA, these are given by (16)-(19), for ULA and UCA, respectively $[16,17]$,

$$
\begin{aligned}
& \mathfrak{R}\{\mathbf{R}(m, n)\}= J_{0}\left(Z_{l}\right)+2 \sum_{k=1}^{\infty} J_{2 k}\left(Z_{l}\right) . \\
& \frac{s^{2}}{s^{2}+4 k^{2}} \cos [2 k \theta] \\
& \mathfrak{J}\{\mathbf{R}(m, n)\}=-2 \sum_{k=1}^{\infty}\left\{J_{2 k+1}\left(Z_{l}\right) \frac{s^{2}\left(1+e^{-s \pi}\right)}{\left[s^{2}+(2 k+1)^{2}\right]\left(1-e^{-s \pi}\right)} .\right. \\
&\sin [(2 k+1) \theta]\} \\
& \mathfrak{R}\{\mathbf{R}(m, n)\}= J_{0}\left(Z_{c}\right)+2 \sum_{k=1}^{\infty} J_{2 k}\left(Z_{c}\right) . \\
& \frac{s^{2}}{s^{2}+4 k^{2}} \cos [2 k(\theta+\alpha)] \\
& \mathfrak{J}\{\mathbf{R}(m, n)\}=-2 \sum_{k=1}^{\infty}\left\{J_{2 k+1}\left(Z_{c}\right) \frac{s^{2}\left(1+e^{-s \pi}\right)}{\left[s^{2}+(2 k+1)^{2}\right]\left(1-e^{-s \pi}\right)} .\right. \\
&\sin [(2 k+1)(\theta+\alpha)]\}
\end{aligned}
$$

where $Z_{l}=2 \pi(m-n) d / \lambda$ and $Z_{c}$ and $\alpha$ are defined in [11]. And $J_{n}($.$) is the Bessel function of first kind and of$ order $n$.

A convenient approach to comparing the two systems is to calculate their respective channel capacities. This provides a comparison that is independent of the specific system details (such as modulation, coding, frame/block size, etc.). The Shannon capacity bound is very well-known. However, it does not always provide a good measure of performance of practical systems, because of the underlying assumptions on infinite block lengths (which imply infinite delay) and error probabilities approaching zero. Practical wireless systems operate with finite, often 
quite short, block lengths and are designed for small but non-vanishing error probabilities. Their performance, especially in the presence of correlated fading, is often worse than predicted by the Shannon bound.

Outage capacity has been used by number of authors, for example in [8] and the references therein, in an attempt to provide a more realistic characterization of performance of wireless communication systems than is provided by Shannon capacity. In these works, the capacity is treated as a random variable, being a function of randomly varying signal-to-noise ratio. They define the outage capacity $C_{\text {out }}(p)$ with probability $p$. This capacity has the intuitive interpretation as the highest transmission rate that can be sustained with probability $(1-p)$. The random capacity of a transmit diversity system is given by,

$$
C=\log _{2}\left(1+\frac{S N R}{2 M} \gamma\right)
$$

The pdf $f(C)$ of the capacity can be computed from the pdf of $\gamma$ using standard random variable transformation and can be given by [8],

$$
f(C)=\frac{2^{C+1} M \log 2}{S N R} f\left(\left(2^{C}-1\right) \frac{2 M}{S N R}\right)
$$

The outage capacity $C_{\text {out }}(p)$ for probability $p$ is given by

$$
p=\int_{-\infty}^{C_{o u t}(p)} f(C) d C
$$

For the case of a beamformer with weight vector $\mathrm{W}=$ $\left[w_{1}, \ldots, w_{M}\right]$, assumed to have unit-norm. The snr at the beamformer output is

$$
s n r=S N R|x|^{2}=S N R\left|\sum_{m=1}^{M} w_{m} a_{m}\right|^{2}
$$

where $x$ is a zero-mean complex Gaussian-random variable with variance $\mathrm{g}=\mathbf{W}^{\mathrm{H}} \mathbf{R W}$, with $\mathbf{R}$ being covariance matrix of the channel response vector $\mathbf{a}$. In the fully correlated case, we can write (23), as

$$
\text { snr }=M \frac{S N R}{2} \gamma ; \text { where, } \gamma=2|a|^{2}
$$

where $a$ is a complex Gaussian variable with zero-mean and unit variance. Therefore, the pdf of the capacity of the beamforming system is given by [8],

$$
f(C)=\frac{2^{C+1} M \log 2}{M S N R} f\left(\left(2^{C}-1\right) \frac{2}{M S N R}\right)
$$

\section{Simulation Results}

The notation in the following figures are given as, TD-U, TD-G, and TD-L which stands for transmit diversity sys- tem with uniform AOA, transmit diversity with truncated Gaussian AOA, and transmit diversity with truncated Laplacian AOA, respectively.

In Figure 2, a comparison is shown at angle spread $(\sigma$ $=3^{\circ}$ ). At $2 \%$ outage probability the performance of TD$\mathrm{U}$ and BF (fully correlated case) is same as concluded by [8] (see Figure 8), but that is not the case with TD-G and TD-L as can be seen from Figure $2[9,10]$. A similar comparison is also plotted when the base station uses UCA. In this case, the performance of TD-U and BF (fully correlated case) is almost same, but inferior to the case when ULA used at the base station. It is clear from the Figure $\mathbf{2}$ and Figure 3 that the transmit diversity systems do not perform well at smaller angle spread, especially for truncated Gaussian AOA.

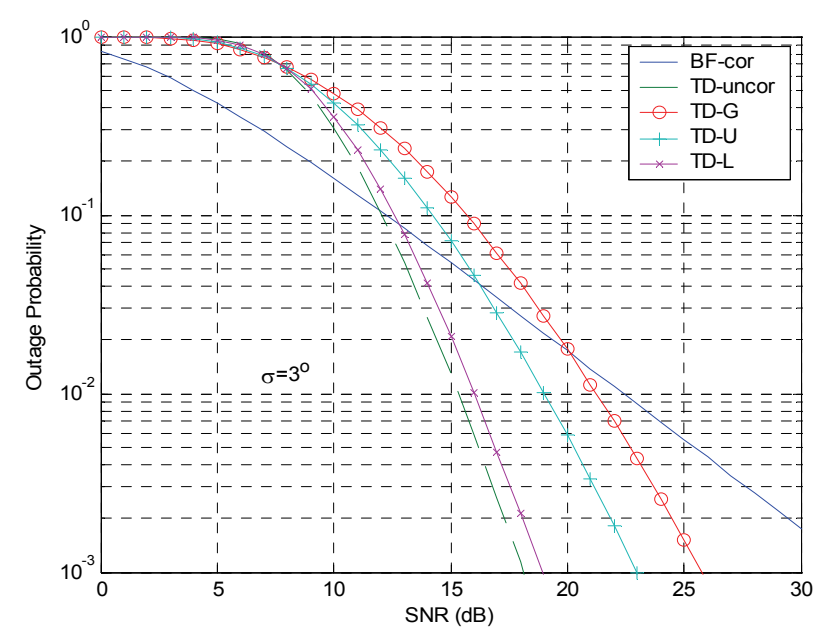

Figure 2. Outage probability for Transmit diversity and beamforming systems for ULA; mean AOA $\theta=0^{\circ}, \sigma=3^{\circ}$, $C_{\text {out }}(p)=3, M=4$.

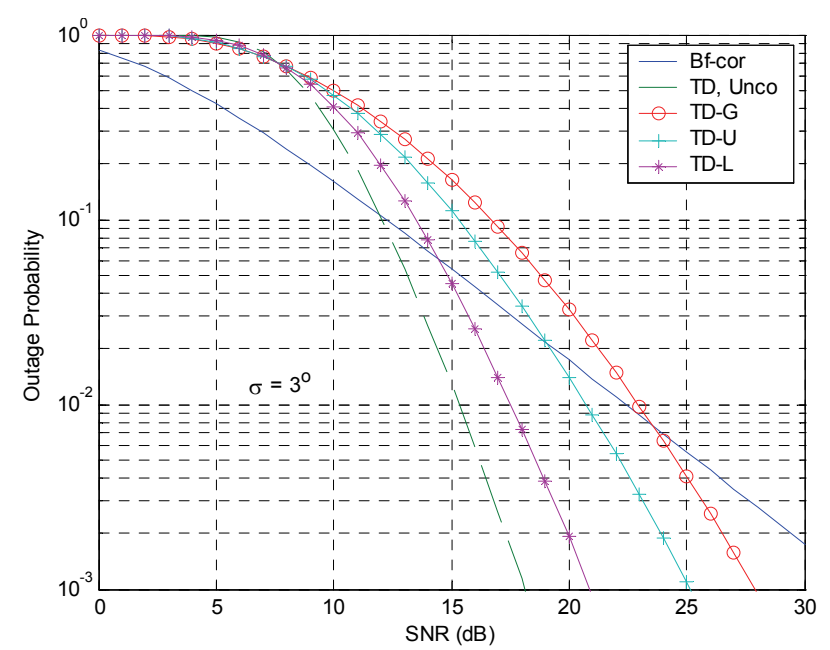

Figure 3. Outage probability for Transmit diversity and beamforming systems for UCA; mean AOA $\theta=0^{\circ}, \sigma=3^{\circ}$, $C_{\text {out }}(p)=3, M=4$. 
To see the effect for wider range of angle spreads, the plot of probability distribution function (pdf) for several values of angle spread using (6), and (12)-(13) are shown in Figures 4 and 5 of [10], where it can be observed that, for a small angle spread of $\left(\sigma=1^{\circ}\right)$, the TD-G curve tends to reach the $\chi_{2}^{2}$ distribution curve, while for moderate angle spread $\left(\sigma=7^{\circ}\right)$, it tends to approach $\chi_{2 M}^{2}$. This is due to reason that, in the case of uncorrelated fading $\mathbf{R}=I, \lambda_{m}=1$, in which case $\gamma$ has a $\chi_{2 M}^{2}$ distribution, while in the fully correlated case, $\mathbf{R}$ has rank one and $\lambda_{1}=M$, while $\lambda_{m}=0$ for $m>1$, in that case, $\gamma / M$ has $\chi_{2}^{2}$ distribution as can be observed from Figure 5 of [10].

Figures 4 and 5 , show the outage capacity curve as a function of $S N R$ of transmit diversity system at $p=2 \%$, for several values of angle spread. In Figure 4, a comparison for TD-U is given for ULA and UCA for two values of angle spread. We can see that, base station with ULA configuration provides higher capacity than that of UCA. Specifically, at $\sigma=7^{\circ}$ and $C=3 \mathrm{bits} / \mathrm{s} / \mathrm{Hz}, \mathrm{UCA}$ is $2 \mathrm{~dB}$ inferior to ULA. Similarly for TD-L and TD-G a comparison is also plotted in Figure 5, for ULA and UCA for $\sigma=7^{\circ}$. It can be noted that, TD-L provides higher capacity than the other two energy distributions.

Figure 6, depicts a plot of spatial correlation as function of antenna separation for ULA for two values of angle spread that is for $\sigma=3^{\circ}$ and $\sigma=7^{\circ}$. Figure 6 also gives sufficient justification that the truncated Laplacian AOA energy distribution gives lower correlation as compared to other energy distributions.

Figure 7, shows the outage capacity curve as a function of SNR for TD-U for several values of central AOA at $p=2 \%$, and $\sigma=3^{\circ}$. It can be seen from the Figure 7 that the central AOA can have significant impact on the performance. Specifically, at $\theta=0^{\circ}$ and $90^{\circ}$ TD-U provides lower capacity values than other central AOA. Only UCA and uniform AOA energy distribution is considered. Similar conclusion can be made for other two energy dis-

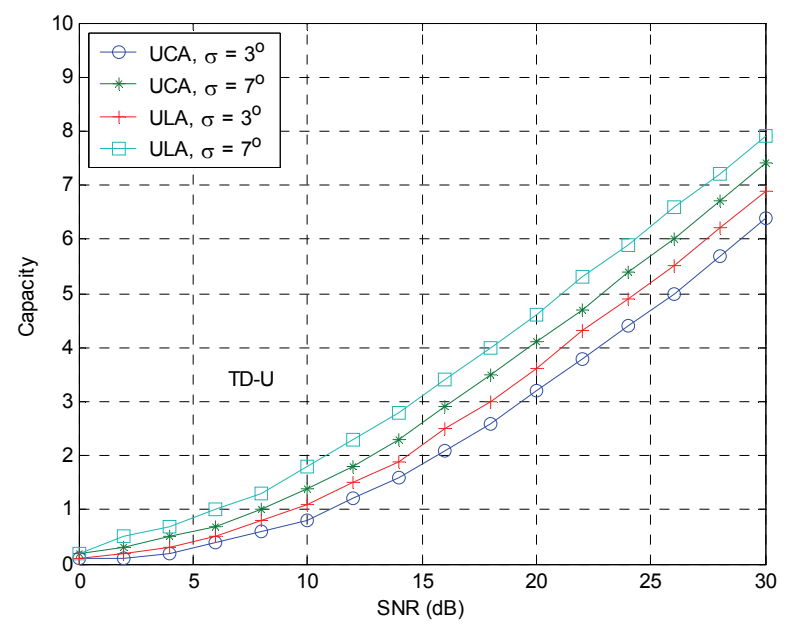

Figure 4. Outage capacity for Transmit diversity for ULA/ UCA with uniform AOA; $\theta=0^{\circ}, M=4, p=2 \%$. tributions.

Based on the above results, we can conclude that the transmit diversity system having ULA or UCA at the base station with truncated Laplacian AOA, perform better than uniform and truncated Gaussian AOA, even at smaller angle spreads. We also show that ULA provide higher capacity than UCA for transmit diversity systems. This conclusion is in consistent with the results reported in [19] for the indoor clustered channels for MIMO communications.

\section{Conclusions}

In this paper, we presented a comparative analysis of transmit diversity system and transmit beamforming for the downlink of wireless communication system, using outage capacity as performance measure. We examined the

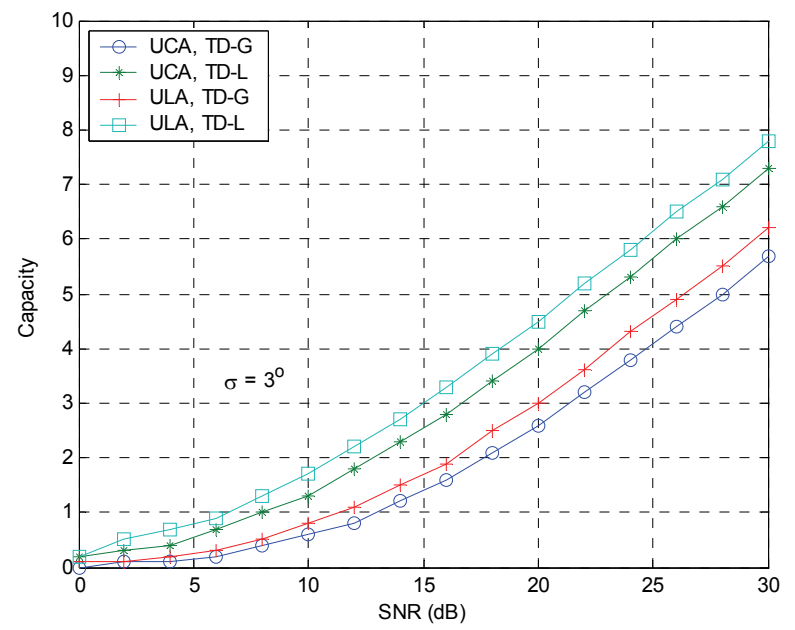

Figure 5. Outage capacity for Transmit diversity for ULA/ UCA; mean AOA $\theta=0^{\circ}, \sigma=3^{\circ}, M=4, p=2 \%$.

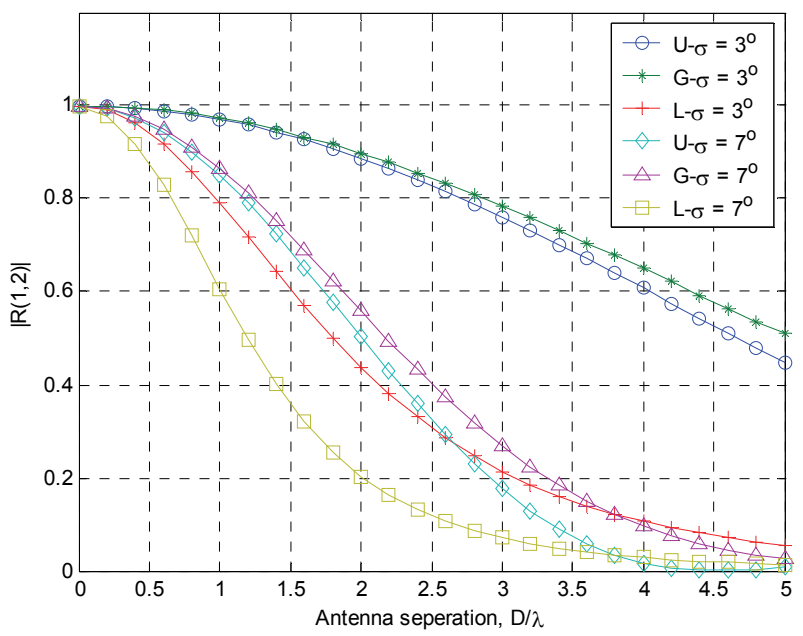

Figure 6. Spatial envelop correlation between antenna 1 and 2 for ULA; mean AOA $\theta=0^{\circ}, M=4$. 


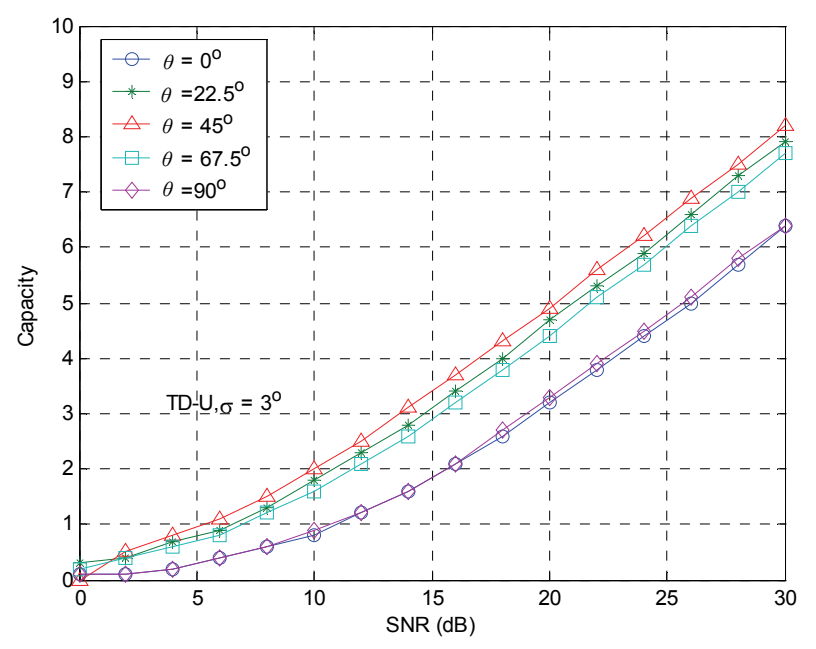

Figure 7. Outage capacity for Transmit diversity for UCA with uniform AOA; $\sigma=3^{\circ}, M=4, p=2 \%$.

effect of angle of arrival (AOA) distributions, (i.e., uniform, truncated Gaussian, and truncated Laplacian), on the two systems under correlated fading for several values of angle spreads. We also examine the array configuration, such as uniform linear array and uniform circular array for these systems. Numerical results show that the transmit diversity system using linear arrays or circular arrays do not perform well at small angle spreads. In the extreme case, where fading is completely correlated, the transmit diversity performs no better than a single antenna. Results also show that the transmit diversity system using arrays (ULA or UCA) with truncated Laplacian AOA, performs better even at smaller angle spreads as compared to other energy distributions. Further, ULA seems to be a preferable configuration to UCA for transmit diversity systems.

\section{References}

[1] J. H. Winters, "The Diversity Gain of Transmit Diversity Technique in Wireless Systems with Rayleigh Fading," IEEE Transactions on Vehicular Technology, Vol. 47, No. 4, 1998, pp. 119-132.

[2] S. Zhou and G. B. Giannakis, "Optimal Transmitter Eigen-Beamforming and Space Time Block Coding Based on Channel Correlations," IEEE Transactions on Information Theory, Vol. 49, No. 7, 2003, pp. 1673-1690.

[3] C. Chauah, D. Tse, J. Kahn and R. Valenzuala, "Capacity Scaling in MIMO Wireless Systems under Correlated Fading," IEEE Transactions on Information Theory, Vol. 48, No. 3, 2002, pp. 637-650.

[4] S. Siwamogsatham and M. P. Fitz, "Robust Space-Time Codes for Correlated Rayleigh Fading Channels," IEEE Transactions on Signal Processing, Vol. 50, No. 10, 2002, pp. 2408-2416.

[5] E. A. Jorswieck and A. Sezgin, "Impact of Spatial Correlation on the Performance of Orthogonal Space-Time Block Codes," IEEE Communications Letters, Vol. 8, No. 1, 2004, pp. 21-23.
[6] C. Rensburg and B. Friedlander, 'Transmit Diversity for Arrays in Correlated Rayleigh Fading," IEEE Transactions on Vehicular Technology, Vol. 53, No. 6, 2004, pp. 1726-1734.

[7] K. I. Pedersen, P. E. Mogensen and B. H. Fleury, "Power Azimuth Spectrum in Outdoor Environments," IEE Electronics Letters, Vol. 33, No. 18, 1997, pp. 1583-1584.

[8] B. Friedlander and S. Scherzer, "Beamforming versus Transmit Diversity in the Downlink of Cellular Communication System," IEEE Transactions on Vehicular Technology, Vol. 53, No. 4, 2004, pp. 1023-1034.

[9] A. W. Umrani and V. K. Dubey, "Effect of Angle of Arrival on Transmit Diversity and Beamforming Systems under Correlated Fading," IEE Electronics Letters, Vol. 41, No. 6, 2005, pp. 293-294.

[10] A. W. Umrani and V. K. Dubey, "Effect of Angle of Arrival on Transmit Diversity and Beamforming Systems under Correlated Fading," IEEE Sarnoff Symposium on Advances in Wired and Wireless Communication, Princeton, 18-19 April 2005, pp. 109-112.

[11] J.-A. Tsai, M. Buehrer and B. D. Woerner, "BER Performance of a Uniform Circular Array versus a Uniform Linear Array in a Mobile Radio Environment," IEEE Transactions on Wireless Communications, Vol. 3, No. 3, 2004, pp. 695-700.

[12] N. L. Johnson, S. Kotz and N. Balakrishnan, "Continuous Univariate Distributions," Wiley Series, Hoboken, Vol. 1, 1994.

[13] J. Salz and J. H. Winters, "Effect of Fading Correlation on Adaptive Arrays in a Digital Mobile Radio," IEEE Transactions on Vehicular Technology, Vol. 43, No. 4, 1994, pp. 1049-1057.

[14] A. W. Umrani and V. K. Dubey, "Correction to BER Performance of a Uniform Circular Array versus a Uniform Linear Array in a Mobile Radio Environment," IEEE Transactions on Wireless Communications, Vol. 5, No. 4, 2006, p. 732.

[15] J.-A. Tsai, M. Buehrer and B. D. Woerner, "The Impact of AOA Energy Distribution on Spatial Fading Correlation of Linear Antenna Arrays," Proceedings of IEEE 55th Vehicular Technology Conference, Birmingham, Vol. 2, 6-9 May 2002, pp. 933-937.

[16] J.-A. Tsai, M. Buehrer and B. D. Woerner, "Spatial Fading Correlation Function of Circular Antenna Arrays with Laplacian Energy Distribution," IEEE Communications Letters, Vol. 6, No. 5, 2002, pp. 178-180.

[17] X. Li and Z. P. Nei, "Comments on Spatial Fading Correlation of Circular Antenna Arrays Laplacian Energy Distribution," IEEE Communications Letters, Vol. 8, No. 5, 2004, p. 295.

[18] Y. Wang, Z. Zhou and J. Wang, "Analysis of Spatial Fading Correlation for Different Antenna Arrays," Proceedings of 2004 IEEE Wireless Communications and Networking Conference, Atlanta, 21-25 March 2004, pp. 700705.

[19] A. Forenza and R. W. Heath, Jr., "Impact of Antenna Geometry on MIMO Communication in Indoor Clustered Channels," Proceedings of Antennas and Propagation Society International Symposium, Monterey, 20-26 June 2004, pp. 1700-1703. 